University of New Orleans

ScholarWorks@UNO

$5-1988$

\title{
General analysis and optimization of the four-detector photopolarimeter
}

\author{
R. M.A. Azzam \\ University of New Orleans, razzam@uno.edu
}

I. M. Elminyawi

A. M. El-Saba

Follow this and additional works at: https://scholarworks.uno.edu/ee_facpubs

Part of the Electrical and Electronics Commons, and the Optics Commons

\section{Recommended Citation}

R. M. A. Azzam, I. M. Elminyawi, and A. M. El-Saba, "General analysis and optimization of the four-detector photopolarimeter," J. Opt. Soc. Am. A 5, 681-689 (1988)

This Article is brought to you for free and open access by the Department of Electrical Engineering at ScholarWorks@UNO. It has been accepted for inclusion in Electrical Engineering Faculty Publications by an authorized administrator of ScholarWorks@UNO. For more information, please contact scholarworks@uno.edu. 


\title{
General analysis and optimization of the four-detector photopolarimeter
}

\author{
R. M. A. Azzam, I. M. Elminyawi, and A. M. El-Saba \\ Department of Electrical Engineering, University of New Orleans, Lakefront, New Orleans, Louisiana 70148
}

Received November 5, 1987; accepted January 13, 1988

\begin{abstract}
The four-detector photopolarimeter (FDP) is analyzed for an arbitrary spatial configuration and any reflection characteristics $\left(r_{i}, \psi_{i}, \Delta_{i}\right)$ of the first three detectors. The instrument matrix $\mathbf{A}$, which relates the output signal vector $\mathbf{I}$ to the input Stokes vector $\mathbf{S}$ by $\mathbf{I}=\mathbf{A S}$, and its determinant are derived explicitly. The essential condition that $\mathbf{A}$ be nonsingular (det $\mathbf{A} \neq 0$ ) is satisfied in general with uncoated absorbing detector surfaces, assuming that the plane of incidence (POI) is rotated between successive reflections by other than $90^{\circ}$. Therefore no special coatings on the detectors are required, and a thin dielectric (e.g., thermal oxide) layer would suffice. The differential reflection phase shift $\Delta$ is unrestricted for the first and third detectors and has optimum values of $\pm 90^{\circ}$ for the second. The optimum rotation angles of the POI are $\pm 45^{\circ}$ and $\pm 135^{\circ}$. The optimum values of the surface parameter $\psi$ are $27.37^{\circ}, 22.5^{\circ}$ or $67.5^{\circ}$, and 0 or $90^{\circ}$ for the first, second, and third reflections, respectively. The following topics are also considered: (1) the partition of energy among detectors, (2) the effect of tilting the last detector, (3) operation of the FDP over a broadband spectral range, (4) choice of the light-beam path, and (5) calibration.
\end{abstract}

\section{INTRODUCTION}

The most general state of (partial elliptical) polarization of a beam of monochromatic or quasi-monochromatic light, as represented by the four Stokes parameters, ${ }^{1}$ can be measured by an arrangement of four photodetectors and no other optical elements, as was demonstrated recently both theoretically and experimentally. ${ }^{2,3}$ In this four-detector photopolarimeter (FDP) (Fig. 1), the light beam is relayed from one detector to the next by partial specular reflection at oblique incidence, and the last detector absorbs substantially all the remaining radiation that impinges upon it at or near normal incidence. Each detector $\mathrm{D}_{k}$ generates an electrical signal $i_{k}(k=0,1,2,3)$ proportional to the fraction of the radiation that it absorbs. Because such a fraction is a certain linear combination of the four Stokes parameters of incident light, the four outputs thus developed form a $4 \times 1$

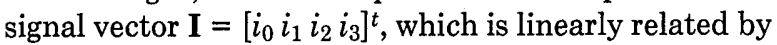

$$
\mathbf{I}=\mathbf{A} \mathbf{S}
$$

to the input Stokes vector $\mathbf{S}=\left[S_{0} S_{1} S_{2} S_{3}\right]^{t}$. ( $t$ indicates the transpose.) Consequently, $\mathbf{S}$ is obtained by

$$
\mathbf{S}=\mathbf{A}^{-1} \mathbf{I} \text {. }
$$

$A$ is a $4 \times 4$ real matrix, called the instrument matrix, that is characteristic of the FDP at a given wavelength.

In this paper we provide a general analysis of the FDP. In particular, the instrument matrix $\mathbf{A}$ is determined for any configuration of the four detectors with arbitrary surface parameters $r_{m}, \psi_{m}$, and $\Delta_{m}(m=0,1,2)$ of the first three detectors and arbitrary rotations $\alpha_{1}$ and $\alpha_{2}$ between the second and first and between the third and second planes of incidence, respectively. $r_{m}$ is the reflectance of the $m$ th detector surface for incident unpolarized or circularly polarized light, and $\tan \psi_{m} \exp \left(j \Delta_{m}\right)=r_{p m} / r_{s m}$ is the ratio of the complex reflection coefficients of the surface for the $p$ and $s$ linear polarizations parallel and perpendicular to the local plane of incidence, respectively. The unambiguous determination of all four Stokes parameters requires that $\mathbf{A}$ be nonsingular, or $\operatorname{det} \mathbf{A} \neq 0$. The singularity conditions are established in detail. Optimum parameters that maximize $|\operatorname{det} \mathbf{A}|$ are also derived. We also consider (1) the partitioning of energy among the four detectors; (2) the effect of tilting the last detector; (3) operating the instrument over a wide spectral range, using Si detectors; (4) light paths; and (5) determination of $\mathbf{A}$ by calibration.

\section{THE EXPLICIT INSTRUMENT MATRIX A}

The Stokes vector of the incident light to be measured and the Stokes vectors of the light reflected from the surfaces of photodetectors $\mathrm{D}_{0}, \mathrm{D}_{1}$, and $\mathrm{D}_{2}$ are

$$
\begin{aligned}
\mathbf{S} & =\mathbf{S}, \\
\mathbf{S}^{(0)} & =\mathbf{M}_{0} \mathbf{S}, \\
\mathbf{S}^{(1)} & =\mathbf{M}_{1} \mathbf{R}_{1} \mathbf{M}_{0} \mathbf{S}, \\
\mathbf{S}^{(2)} & =\mathbf{M}_{2} \mathbf{R}_{2} \mathbf{M}_{1} \mathbf{R}_{1} \mathbf{M}_{0} \mathbf{S},
\end{aligned}
$$

respectively, where

$$
\begin{aligned}
& \mathbf{M}_{l}\left[\begin{array}{cccc}
1 & -\cos 2 \psi_{l} & 0 & 0 \\
-\cos 2 \psi_{l} & 1 & 0 & 0 \\
0 & 0 & \sin 2 \psi_{l} \cos \Delta_{l} & \sin 2 \psi_{l} \sin \Delta_{l} \\
0 & 0 & -\sin 2 \psi_{l} \sin \Delta_{l} & \sin 2 \psi_{l} \cos \Delta_{l}
\end{array}\right]
\end{aligned}
$$

is the reflection Mueller matrix ${ }^{4}$ of the $l$ th detector and 


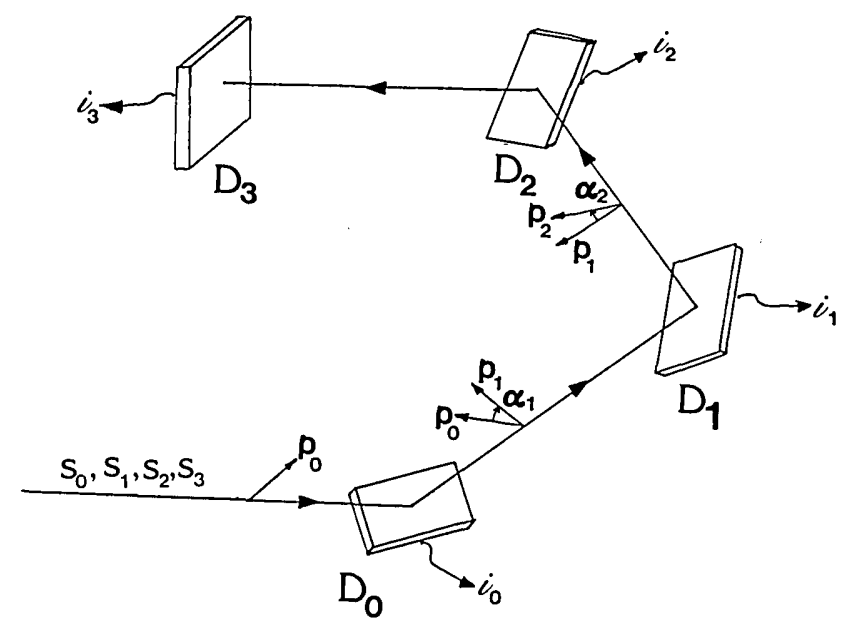

Fig. 1. Diagram of the FDP. The surfaces of photodetectors $D_{0}$, $D_{1}$, and $D_{2}$ are partially specularly reflecting, whereas that of $D_{3}$ is substantially totally absorbing. The four output currents $i_{0}, i_{1}, i_{2}$, and $i_{3}$ determine the input Stokes parameters $S_{0}, S_{1}, S_{2}$, and $S_{3}$. $p_{n}$ is a transverse reference polarization direction parallel to the $n$th plane of incidence. $\alpha_{1}$ and $\alpha_{2}$ are the rotation angles of the plane of incidence.

$$
\mathbf{R}_{l}=\left[\begin{array}{cccc}
1 & 0 & 0 & 0 \\
0 & \cos 2 \alpha_{l} & \sin 2 \alpha_{l} & 0 \\
0 & -\sin 2 \alpha_{l} & \cos 2 \alpha_{l} & 0 \\
0 & 0 & 0 & 1
\end{array}\right]
$$

is the rotation Mueller matrix ${ }^{4}$ that describes the effect of rotation of the plane of incidence between successive reflections. [The significance of adding the first of Eqs. (3) becomes apparent in what follows.]

The light flux of the beam along its segmented path is given by $S_{0}, S_{0}{ }^{(0)}, S_{0}^{(1)}$, and $S_{0}^{(2)}$, which are the first elements of the Stokes vectors $\mathbf{S}, \mathbf{S}^{(0)}, \mathbf{S}^{(1)}$, and $\mathbf{S}^{(2)}$, respectively. Formally, each flux is obtained by premultiplying both sides of Eq. (3) by the row vector

$$
\Gamma=\left[\begin{array}{llll}
1 & 0 & 0 & 0
\end{array}\right] \text {. }
$$

It is convenient to combine these fluxes into a single light vector

$$
\mathbf{L}=\left[\begin{array}{c}
S_{0} \\
S_{0}{ }^{(0)} \\
S_{0}{ }^{(1)} \\
S_{0}{ }^{(2)}
\end{array}\right] .
$$

$\mathbf{L}$ is linearly related to the input Stokes vector by

$$
\mathbf{L}=\mathbf{F S} \text {, }
$$

where $\mathbf{F}$ can be expressed in terms of its rows as follows:

$$
\mathbf{F}=\left[\begin{array}{l}
\mathbf{F}_{0} \\
\mathbf{F}_{1} \\
\mathbf{F}_{2} \\
\mathbf{F}_{3}
\end{array}\right]=\left[\begin{array}{c}
\Gamma \\
\Gamma \mathbf{M}_{0} \\
\Gamma \mathbf{M}_{1} \mathbf{R}_{1} \mathbf{M}_{0} \\
\Gamma \mathbf{M}_{2} \mathbf{R}_{2} \mathbf{M}_{1} \mathbf{R}_{1} \mathbf{M}_{0}
\end{array}\right] .
$$

By the definition of $\Gamma$ [Eq. (6)], it is obvious that the second, third, and fourth rows of $\mathbf{F}\left(\mathbf{F}_{1}, \mathbf{F}_{2}\right.$, and $\left.\mathbf{F}_{3}\right)$ are the first rows of the matrices $\mathbf{M}_{0}, \mathbf{M}_{1} \mathbf{R}_{1} \mathbf{M}_{0}$, and $\mathbf{M}_{2} \mathbf{R}_{2} \mathbf{M}_{1} \mathbf{R}_{1} \mathbf{M}_{0}$, respective- ly. The first two rows of $\mathbf{F}$ are obtained by simple inspection, and the remaining two are obtained by performing the matrix multiplications that appear in $\mathbf{F}_{2}$ and $\mathbf{F}_{3}$, using the explicit forms of $\mathbf{M}_{l}$ and $\mathbf{R}_{l}$ in Eqs. (4) and (5). The result is

$$
\mathbf{F}=\left[\begin{array}{cccc}
1 & 0 & 0 & 0 \\
f_{10} & f_{11} & 0 & 0 \\
f_{20} & f_{21} & f_{22} & f_{23} \\
f_{30} & f_{31} & f_{32} & f_{33}
\end{array}\right],
$$

where

$$
\begin{aligned}
& f_{10}=r_{0} \text {, } \\
& f_{11}=-r_{0} \cos 2 \psi_{0} \text {, } \\
& f_{20}=r_{0} r_{1}\left(1+\cos 2 \psi_{0} \cos 2 \psi_{1} \cos 2 \alpha_{1}\right) \text {, } \\
& f_{21}=-r_{0} r_{1}\left(\cos 2 \psi_{0}+\cos 2 \psi_{1} \cos 2 \alpha_{1}\right) \text {, } \\
& f_{22}=-r_{0} r_{1}\left(\sin 2 \psi_{0} \cos \Delta_{0} \cos 2 \psi_{1} \sin 2 \alpha_{1}\right) \text {, } \\
& f_{23}=-r_{0} r_{1}\left(\sin 2 \psi_{0} \sin \Delta_{0} \cos 2 \psi_{1} \sin 2 \alpha_{1}\right) \text {, } \\
& f_{30}=r_{0} r_{1} r_{2}\left(1+\cos 2 \psi_{0} \cos 2 \psi_{1} \cos 2 \alpha_{1}\right. \\
& +\cos 2 \psi_{1} \cos 2 \psi_{2} \cos 2 \alpha_{2} \\
& +\cos 2 \psi_{0} \cos 2 \psi_{2} \cos 2 \alpha_{1} \cos 2 \alpha_{2} \\
& -\cos 2 \psi_{0} \sin 2 \psi_{1} \cos \Delta_{1} \cos 2 \psi_{2} \sin 2 \alpha_{1} \sin 2 \alpha_{2} \text { ), } \\
& f_{31}=-r_{0} r_{1} r_{2}\left(\cos 2 \psi_{0}+\cos 2 \psi_{1} \cos 2 \alpha_{1}\right. \\
& +\cos 2 \psi_{0} \cos 2 \psi_{1} \cos 2 \psi_{2} \cos 2 \alpha_{2} \\
& +\cos 2 \psi_{2} \cos 2 \alpha_{1} \cos 2 \alpha_{2} \\
& \left.-\sin 2 \psi_{1} \cos \Delta_{1} \cos 2 \psi_{2} \sin 2 \alpha_{1} \sin 2 \alpha_{2}\right) \text {, } \\
& f_{32}=-r_{0} r_{1} r_{2}\left(\sin 2 \psi_{0} \cos \Delta_{0} \cos 2 \psi_{1} \sin 2 \alpha_{1}\right. \\
& +\sin 2 \psi_{0} \cos \Delta_{0} \cos 2 \psi_{2} \sin 2 \alpha_{1} \cos 2 \alpha_{2} \\
& +\sin 2 \psi_{0} \cos \Delta_{0} \sin 2 \psi_{1} \cos \Delta_{1} \cos 2 \psi_{2} \cos 2 \alpha_{1} \sin 2 \alpha_{2} \\
& -\sin 2 \psi_{0} \sin \Delta_{0} \sin 2 \psi_{1} \sin \Delta_{1} \cos 2 \psi_{2} \sin 2 \alpha_{2} \text { ), } \\
& f_{33}=-r_{0} r_{1} r_{2}\left(\sin 2 \psi_{0} \sin \Delta_{0} \cos 2 \psi_{1} \sin 2 \alpha_{1}\right. \\
& +\sin 2 \psi_{0} \sin \Delta_{0} \cos 2 \psi_{2} \sin 2 \alpha_{1} \cos 2 \alpha_{2} \\
& +\sin 2 \psi_{0} \cos \Delta_{0} \sin 2 \psi_{1} \sin \Delta_{1} \cos 2 \psi_{2} \sin 2 \alpha_{2} \\
& \left.+\sin 2 \psi_{0} \sin \Delta_{0} \sin 2 \psi_{1} \cos \Delta_{1} \cos 2 \psi_{2} \cos 2 \alpha_{1} \sin 2 \alpha_{2}\right) \text {. }
\end{aligned}
$$

The electrical output signal of each detector is proportional to the light flux that it absorbs, which is the difference between the incident and reflected fluxes, so that

$$
\begin{aligned}
& i_{0}=k_{0}\left(S_{0}-S_{0}{ }^{(0)}\right), \\
& i_{1}=k_{1}\left(S_{0}{ }^{(0)}-S_{0}{ }^{(1)}\right), \\
& i_{2}=k_{2}\left(S_{0}{ }^{(1)}-S_{0}{ }^{(2)}\right), \\
& i_{3}=k_{3} S_{0}{ }^{(2)},
\end{aligned}
$$

where $k_{n}$ is the responsivity of the $n$th detector and includes any postdetection amplification factor. The last element of 
Eqs. (12) is consistent with the assumption that the last detector is nonreflecting or totally absorptive. Equations (12) can be cast in matrix form as

$$
\mathbf{I}=\mathbf{K D L},
$$

where $\mathbf{K}$ is the diagonal responsivity matrix,

$$
\begin{aligned}
& \mathbf{K}=\left[\begin{array}{cccc}
k_{0} & 0 & 0 & 0 \\
0 & k_{1} & 0 & 0 \\
0 & 0 & k_{2} & 0 \\
0 & 0 & 0 & k_{3}
\end{array}\right], \\
& \mathbf{D}=\left[\begin{array}{rrrr}
1 & -1 & 0 & 0 \\
0 & 1 & -1 & 0 \\
0 & 0 & 1 & -1 \\
0 & 0 & 0 & 1
\end{array}\right],
\end{aligned}
$$

and $\mathbf{L}$ is the light vector of Eq. (7). Clearly, $\mathbf{D}$ is a convenient matrix that determines the detected flux differences, when it premultiplies the light vector L. By combining Eqs. (8) and (13), we obtain

$$
\mathbf{I}=\text { KDFS. }
$$

Comparing Eq. (16) with Eq. (1) establishes the instrument matrix in the form

$$
\mathbf{A}=\mathbf{K D F},
$$

where $\mathbf{K}, \mathbf{D}$, and $\mathbf{F}$ are given explicitly by Eqs. (14), (15), and (10) and (11), respectively. This completes the task of finding the instrument matrix of the general FDP. [Obviously, there is nothing to gain by further expansion of Eq. (17), considering the appearance of the elements of $\mathbf{F}$ in Eqs. (11).]

\section{SINGULARITIES OF THE INSTRUMENT MATRIX}

From Eq. (2) it is required that $\mathbf{A}^{-1}$ exist for the unambiguous determination of the full Stokes vector $\mathbf{S}$ from the output current vector $\mathbf{I}$. This means that $\mathbf{A}$ must be nonsingular and its determinant, $\operatorname{det} \mathbf{A}$, must be nonzero. Therefore it is essential to calculate $\operatorname{det} \mathbf{A}$. From Eq. (17),

$$
\operatorname{det} \mathbf{A}=(\operatorname{det} \mathbf{K})(\operatorname{det} \mathbf{D})(\operatorname{det} \mathbf{F}) \text {, }
$$

since the determinant of a product of a number of matrices equals the product of the determinants of the individual matrices. From Eqs. (10), (14), and (15), we have

$$
\begin{aligned}
& \operatorname{det} \mathbf{K}=k_{0} k_{1} k_{2} k_{3}, \\
& \operatorname{det} \mathbf{D}=1, \\
& \operatorname{det} \mathbf{F}=f_{11}\left(f_{22} f_{33}-f_{23} f_{32}\right),
\end{aligned}
$$

so that

$$
\operatorname{det} \mathbf{A}=\left(k_{0} k_{1} k_{2} k_{3}\right) f_{11}\left(f_{22} f_{33}-f_{23} f_{32}\right) .
$$

Finally, substitution of $f_{i j}$ from Eqs. (11) into Eq. (20) gives

$$
\begin{aligned}
\operatorname{det} \mathbf{A}= & -\left(k_{0} k_{1} k_{2} k_{3}\right)\left(r_{0}{ }^{3} r_{1}{ }^{2} r_{2}\right)\left(\sin 2 \alpha_{1} \sin 2 \alpha_{2}\right) \\
& \times\left(\sin ^{2} 2 \psi_{0} \cos 2 \psi_{0} \sin 2 \psi_{1} \cos 2 \psi_{1} \cos 2 \psi_{2}\right)\left(\sin \Delta_{1}\right) .
\end{aligned}
$$

It is remarkable that the determinant of the instrument matrix is in the product form of Eq. (21). $\operatorname{det} \mathbf{A}=0$, and $\mathbf{A}$ is singular, if any of the multiplicative terms is zero. It is instructive to group the singularity conditions as follows:

(1) $k_{l}=0, l=0,1,2,3$. If the responsivity of any detector is zero, the corresponding output signal disappears, and a measurement is lost. This can happen, e.g., if a detector fails or if its power supply is disconnected, in which case it becomes a passive reflector producing no output current.

(2) $r_{l}=0$ for $l=0,1,2 . \quad r_{l}=0$ means that the light beam vanishes after the $l$ th detector and is not relayed to subsequent ones, leading to the loss of one or more signals. Making the first three detector surfaces reflective guarantees that this type of singularity will not occur.

(3) The third bracketed term on the right-hand side of Eq. (21), $\sin 2 \alpha_{1} \sin 2 \alpha_{2}$, vanishes if $\alpha_{1}$ or $\alpha_{2}=0,90^{\circ}, 180^{\circ}$. Thus the planes of incidence of two successive reflections must not coincide or be orthogonal. The light path in the FDP can be chosen easily to avoid this singularity once and for all. (Because $\alpha_{1}$ and $\alpha_{2}$ are purely geometrical parameters, characteristic of the light path, this type of singularity is avoidable independent of the wavelength of light.)

(4) The fourth (psi) term in Eq. (21) vanishes if any of the following conditions is satisfied:

$$
\begin{aligned}
& \psi_{0}=0 \text { or } \pi / 2, \\
& \psi_{0}=\pi / 4, \\
& \psi_{1}=0 \text { or } \pi / 2, \\
& \psi_{1}=\pi / 4, \\
& \psi_{2}=\pi / 4 .
\end{aligned}
$$

Recall that, by definition, $\psi$ assumes values in the range $0 \leq$ $\psi \leq \pi / 2$. Equations (22a) and (22c) tell us that the surfaces of the first two detectors must not act as perfect linear polarizers, whereas Eqs. (22b), (22d), and (22e) indicate that none of the surfaces of the first three detectors should reflect the $p$ and $s$ polarizations equally (or function as a reflection retarder). From what we know about the reflection of light from coated and uncoated absorbing surfaces, ${ }^{4}$ these singularities would not occur unless by design. If the detectors are coated by transparent films of thickness of the order of the wavelength of light, these psi singularities may occur at a few discrete wavelengths in broadband spectral applications.

(5) Finally, consider the last term on the right-hand side of Eq. (21). This term, sin $\Delta_{1}$, shows that the successful operation of the FDP as a complete photopolarimeter depends on one essential differential reflection phase shift, $\Delta_{1}$, at the surface of the second detector. The absence of $\Delta_{0}$ and $\Delta_{2}$ from the determinant is notable and indicates that these phase shifts are unrestricted. This makes detector surface design much simpler. Obviously, singularities occur when

$$
\Delta_{1}=0 \text { or } \pi \text {. }
$$

Such phase shifts are typically associated with light reflection from transparent (dielectric) surfaces. Thus the mere fact that a photodetector surface is absorbing guarantees that the delta singularity will not occur. Again, for an opti- 
cally thick coating and over a broad spectral range, Eq. (23) may be satisfied at a few discrete wavelengths.

From the preceding discussion we reach the following important conclusions: (1) With simple precautions the instrument matrix can be made nonsingular over a wide spectral range. This is demonstrated further in Section 6 for a FDP using Si detectors. (2) Operation of the FDP does not. require special thin-film coatings of the detector surfaces because the singularity conditions are avoided readily with uncoated absorbing surfaces.

\section{OPTIMIZATION}

We now seek optimum parameters that make the FDP instrument matrix as far from singular as possible by maximizing the absolute value of a normalized determinant defined by

$$
(\operatorname{det} \mathbf{A})_{n}=\operatorname{det} \mathbf{A} /\left(k_{0} k_{1} k_{2} k_{3}\right)\left(r_{0}{ }^{3} r_{1}^{2} r_{2}\right) .
$$

(1) Rotation angles of the plane of incidence of

$$
\alpha_{1}, \alpha_{2}= \pm 45^{\circ} \text { or } \pm 135^{\circ}
$$

maximize the absolute value of the alpha term of the determinant, $T_{\alpha}=\left|\sin 2 \alpha_{1} \sin 2 \alpha_{2}\right|$, with the maximum value being 1. It should be noted that changing the angles between planes of incidence by $\pm 15^{\circ}$ around the optimum values of Eq. (25) diminishes $T_{\alpha}$ by no more than $25 \%$ [ $\sin (2 \times$ $\left.30^{\circ}\right) \sin \left(2 \times 30^{\circ}\right)=0.75$ ]; therefore the selection of a light path is not critical.

(2) The psi term of the determinant can be broken into a product, $T_{\psi}=T_{\psi_{0}} T_{\psi_{1}} T_{\psi_{2}}$, where $T_{\psi_{0}}=\sin ^{2} 2 \psi_{0} \cos 2 \psi_{0}, T_{\psi_{1}}=$ $1 / 2 \sin 4 \psi_{1}$, and $T_{\psi_{2}}=\cos 2 \psi_{2}$. The maximum of $T_{\psi_{0}}$ occurs when

$$
\frac{\mathrm{d}}{\mathrm{d} \psi_{0}}\left(\sin ^{2} 2 \psi_{0} \cos 2 \psi_{0}\right)=0
$$

which gives

$$
\psi_{0}=1 / 2 \cos ^{-1} \sqrt{1 / 3}=27.37^{\circ},
$$

at which $T_{\psi_{0}}^{\max }=2 / 3 \sqrt{3} . \quad T_{\psi_{1}}$ is maximum (=1/2) when $4 \psi_{1}=\pi /$ 2 or $3 \pi / 2$, which gives

$$
\psi_{1}=\pi / 8=22.5^{\circ}
$$

or

$$
\psi_{1}=3 \pi / 8=67.5^{\circ} .
$$

Finally, $\left|T_{\psi_{2}}\right|$ is maximum (=1) when $2 \psi_{2}=0$ or $\pi$, or

$$
\psi_{2}=0 \text { or } 90^{\circ} \text {. }
$$

The optimum values of $\psi_{2}$ in Eq. (26c) indicate that the third detector surface should, ideally, be polarizing. The overall maximum absolute value of $T_{\psi}$ is $1 / 3 \sqrt{3}$.

(3) The remaining delta term, $T_{\Delta}=\left|\sin \Delta_{1}\right|$, is maximum $(=1)$ when

$$
\Delta_{1}= \pm 90^{\circ},
$$

which corresponds to light incident upon the second detector at a principal angle. ${ }^{5}$

Because a function is stationary at a point of maximum value, small changes of $\psi_{0}, \psi_{1}, \psi_{2}$, and $\Delta_{1}$ around the optimum values given by Eqs. (26) and (27) do not affect the determinant to first order. The maximum absolute value of the overall normalized determinant is

$$
(\operatorname{det} \mathbf{A})_{n}^{\max }=1 / 3 \sqrt{3} \text {. }
$$

\section{PARTITION OF ENERGY AMONG THE FOUR DETECTORS}

An interesting question related to the FDP has to do with the choice of the surface reflectivities $r_{0}, r_{1}$, and $r_{2}$ of the first three detectors. (Recall that $r_{3}=0$; i.e., the last detector is totally absorbing.) This, in turn, determines the partition of input beam energy among the four detectors in the course of polarization measurement.

One meaningful criterion is that of equal weight in the allimportant determinant of the instrument matrix A. From Eq. (21), we see that this is accomplished when

$$
r_{0}{ }^{3}=r_{1}{ }^{2}=r_{2} \text {. }
$$

Equation (29) represents an intuitively desirable constraint on detector surface reflectances. According to Eq. (29) the reflectivity is highest for the first detector and decreases gradually as we go to the second and third detectors. If we were to make a special effort to satisfy Eqs. (29), this would involve control of the angles of incidence and possibly thinfilm coatings on the detector surfaces.

Another criterion for selecting $r_{0}, r_{1}$, and $r_{2}$ is equipartition of energy among the four detectors for incident unpolarized light. (It should be noted that for a given FDP, the division of input radiation power among the four detectors is a function of incident polarization; that is exactly why such polarization can be measured.) The normalized (unit-power) Stokes vector of incident unpolarized light is

$$
\mathbf{S}=\left[\begin{array}{lll}
1 & 0 & 0
\end{array}\right]^{t}
$$

Substitution of this vector into Eq. (8) gives the light vector

$$
\mathbf{L}_{u}=\left[1 f_{10} f_{20} f_{30}\right]^{t},
$$

where the subscript $u$ reminds us of the assumed unpolarized incident radiation. The flux differences absorbed by the four detectors are the elements of the vector

$$
\mathbf{V}=\mathbf{D L}_{u}=\left[\left(1-f_{10}\right)\left(f_{10}-f_{20}\right)\left(f_{20}-f_{30}\right) f_{30}\right]^{t},
$$

where $\mathbf{D}$ is the difference matrix of Eq. (15). Equipartition is achieved by setting every element of $\mathrm{V}$ equal to $1 / 4$ :

$$
\begin{aligned}
& 1-f_{10}=1 / 4, \quad f_{10}-f_{20}=1 / 4, \\
& f_{20}-f_{30}=1 / 4, \quad f_{30}=1 / 4 .
\end{aligned}
$$

Equations (33) simplify to

$$
\begin{aligned}
& f_{10}=3 / 4, \\
& f_{20}=1 / 2, \\
& f_{30}=1 / 4 .
\end{aligned}
$$

From Eqs. (11a) and (34a), we get

$$
r_{0}=3 / 4 \text {, or } 75 \% \text {. }
$$


From Eqs. (11c), (11g), (34b), and (35c), it becomes evident that $r_{1}$ and $r_{2}$, which are required for equipartition, will depend in general on the other system parameters $\alpha_{i}, \psi_{i}$, and $\Delta_{i}$. If the optimum choice $\cos 2 \alpha_{1}=\cos 2 \alpha_{2}=0$ (e.g., $\alpha_{1}=\alpha_{2}$ $=45^{\circ}$ ) is made, which is always possible by the selection of the light path, we get, from Eqs. (11c) and (34b),

$$
r_{1}=2 / 3 \text {, or } 66.67 \% \text {. }
$$

Finally, if we also take $\cos \Delta_{1}=0$ (e.g., $\Delta_{1}=90^{\circ}$ ), which is also optimal, we get, from Eqs. (11g) and (34c),

$$
r_{2}=1 / 2 \text {, or } 50 \% \text {. }
$$

As before, Eqs. (35) may be satisfied by control of incidence angles and detector coatings.

Whereas tailoring the surface reflectances according to one or the other of the foregoing criteria is desirable, the FDP will continue to function satisfactorily as long as a nonnegligible fraction of the incident radiation (e.g., a few percent) reaches the last detector. In this regard, notice that, aside from noise considerations, the detector responsivity and gain can make up for a reduced light level. This is apparent in Eq. (21), where the $k$ product precedes the $r$ product.

\section{EFFECT OF TILTING THE LAST DETECTOR}

In Fig. 1, the last detector $D_{3}$ is shown to intercept the light beam at normal incidence. Because the surface of $D_{3}$ cannot be made totally absorptive (or perfectly antireflective) over a broad spectral range, it is desirable to tilt that detector by a small angle to avoid multiple reflections inside the FDP. The weak residual beam that is reflected by $D_{3}$ is dumped. ${ }^{3}$

If the surface of $D_{3}$ is optically isotropic and planar (coated or uncoated) and is tilted by a small angle $\left(<10^{\circ}\right)$, the reflectance of that surface, $r_{3}$, stays independent of the polarization of incident light. This follows from the stationary property of reflection near normal incidence, which is discussed elsewhere. ${ }^{6}$ Under these conditions, the analysis of Sections 2-4 remains intact, except for the minor substitution of $\left(1-r_{3}\right) k_{3}$ in the place of $k_{3}$ wherever it appears.

In conclusion, the operation of the FDP is unaffected by the small tilt of the last detector that is required to block unwanted optical feedback. (The case of the in-line lightsaving photopolarimeter, ${ }^{7}$ in which the last detector is tilted by a large angle and all detector surfaces are made highly reflective to preserve the power and the direction of the incident light, falls outside the scope of this paper.)

\section{OPERATION OF THE FOUR-DETECTOR PHOTOPOLARIMETER OVER A BROAD SPECTRAL RANGE}

It is important to demonstrate that, for a given set of four detectors and a given light path, the FDP can measure all four Stokes parameters of incident light, if the wavelength is changed over a broad spectral range.

As an example, we assume that the first three photodetectors are identical, windowless, planar Si photodiodes with a passivation $\mathrm{SiO}_{2}$ layer only $10 \mathrm{~nm}$ thick on each detector surface. For simplicity, we also assume that the light beam strikes each detector at the same angle of incidence $\phi$ and

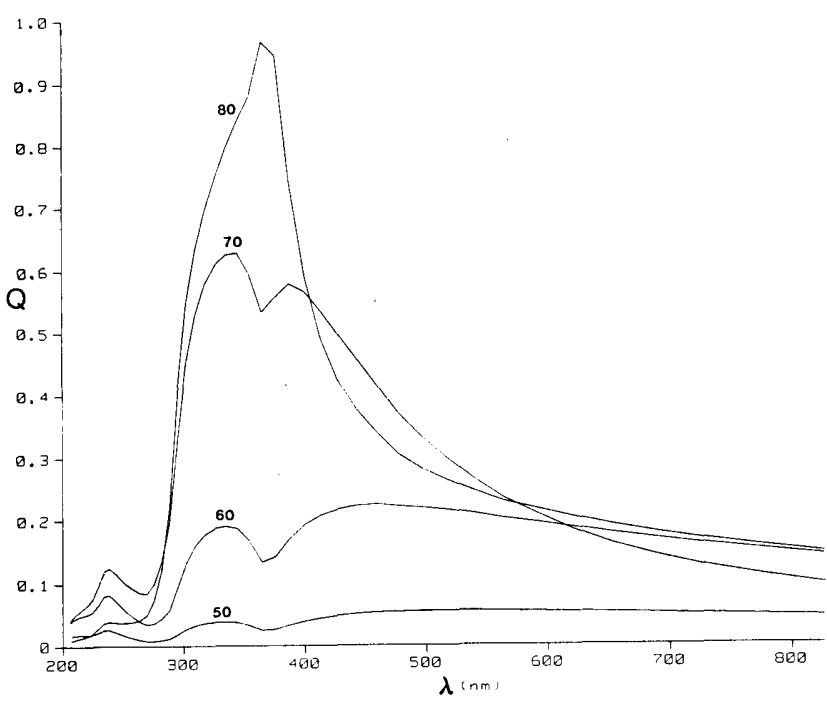

Fig. 2. The quantity $Q(\lambda)$ [Eq. (36)], which is $1 / 8$ of the normalized determinant of the instrument matrix $\mathbf{A}$, versus the wavelength $\lambda$ for a FDP that uses identical Si detectors $D_{0}, D_{1}$, and $D_{2}$, each of which is coated by a thin $(10-\mathrm{nm}) \mathrm{SiO}_{2}$ film and each of which reflects light at the same angle of incidence $\phi$. The four curves correspond to $\phi=50^{\circ}, 60^{\circ}, 70^{\circ}, 80^{\circ}$. The optical properties of $\mathrm{SiO}_{2}$ and Si are taken from Refs. 8 and 9, respectively. Notice that $Q(\lambda)$ $\neq 0$, and hence $\mathbf{A}$ is nonsingular over the entire 207-826-nm spectral range.

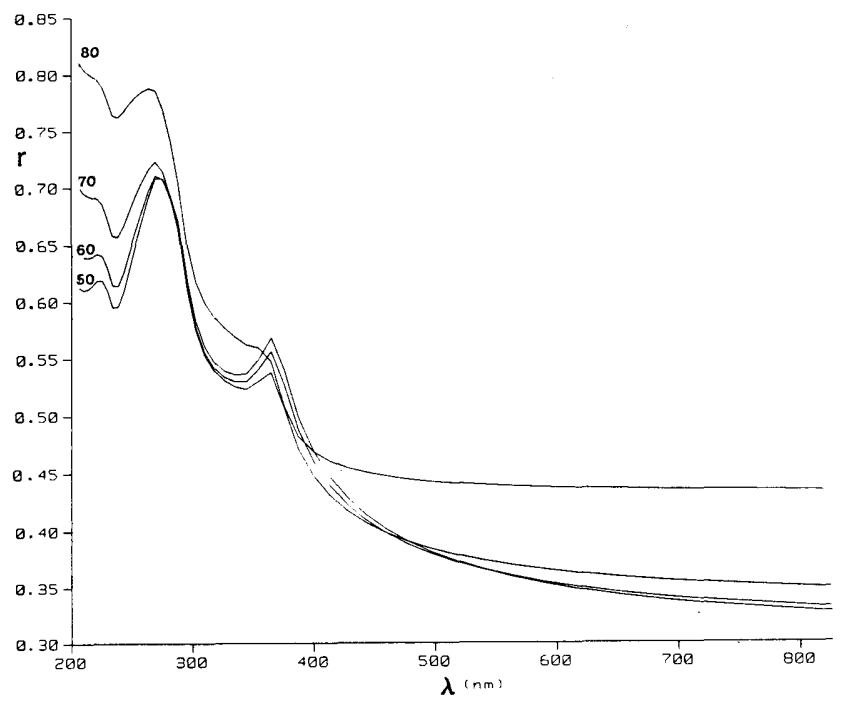

Fig. 3. The unpolarized-light spectral reflectance of a Si detector coated by a 10 -nm $\mathrm{SiO}_{2}$ film at four angles of incidence $\phi=50^{\circ}, 60^{\circ}$, $70^{\circ}, 80^{\circ}$. The optical properties of $\mathrm{SiO}_{2}$ and $\mathrm{Si}$ are taken from Refs. 8 and 9 , respectively.

that the plane of incidence rotates by $\pm 45^{\circ}$ or $\pm 135^{\circ}$ between the first and second and between the second and third reflections (such rotations, $\alpha_{1}$ and $\alpha_{2}$, are optimal, as is discussed in Section 4). From Eqs. (21) and (24), the normalized determinant of the instrument matrix is given by

$$
\pm 8(\operatorname{det} \mathbf{A})_{n}=Q=\sin ^{3}(4 \psi) \sin \Delta
$$

where $\psi=\psi_{0}=\psi_{1}=\psi_{2}$ and $\Delta=\Delta_{1}$.

Figure 2 shows the quantity $Q(\lambda)$ plotted as a function of the wavelength $\lambda$ between 207 and $826 \mathrm{~nm}(1.5-6 \mathrm{eV}$ of 


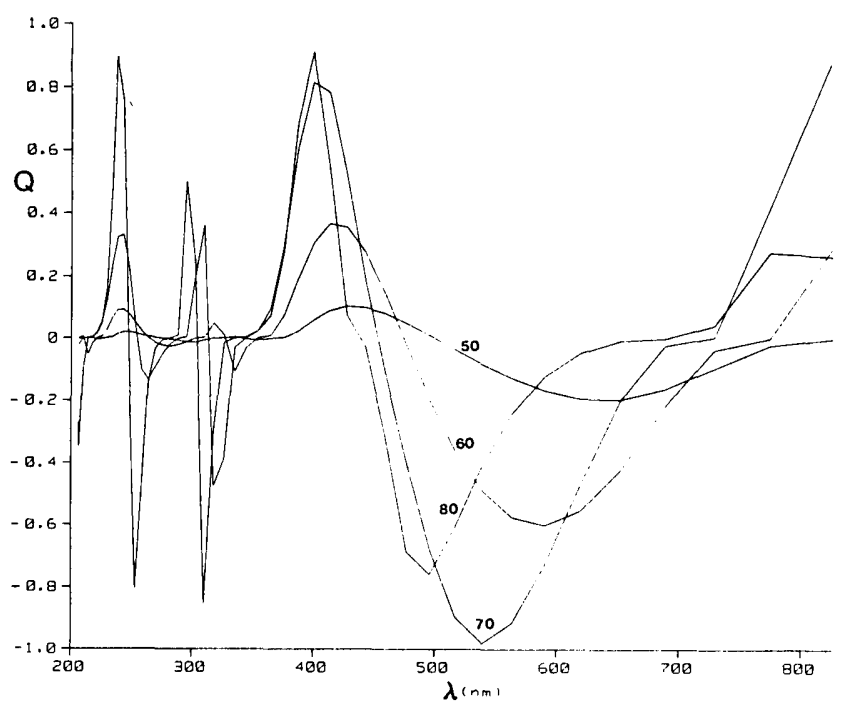

Fig. 4. Same as in Fig. 2 except that the thickness of the $\mathrm{SiO}_{2}$ film is now $200 \mathrm{~nm}$. The oscillatory behavior of $Q(\lambda)$, a consequence of interference in the optically thick film, causes the instrument matrix to be singular (det $\mathbf{A}=0$ ) at a number of discrete wavelengths.

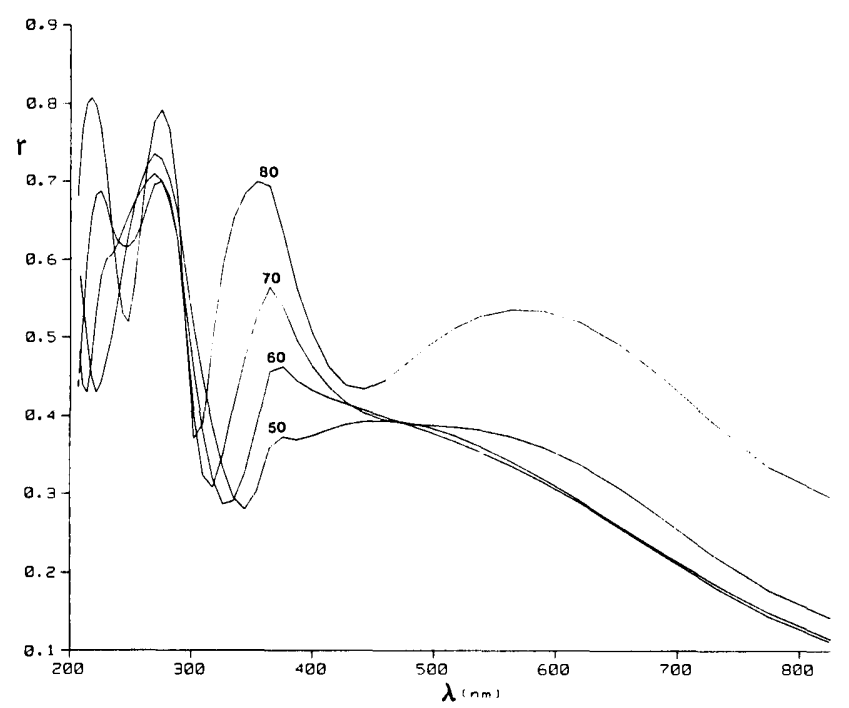

Fig. 5. Same as in Fig. 3, except that the $\mathrm{SiO}_{2}$ film is now $200 \mathrm{~nm}$ thick.

photon energy) at four angles of incidence $\phi=50^{\circ}, 60^{\circ}, 70^{\circ}$, $80^{\circ}$. In this calculation the dispersion of the optical properties of $\mathrm{SiO}_{2}$ and $\mathrm{Si}$ is fully accounted for by using the complex dielectric function (or refractive index) data given in Refs. 8 and 9 , respectively.

Two important conclusions are immediately drawn from Fig. 2: (1) $Q \neq 0$, and $\mathbf{A}$ is nonsingular over the entire spectral range. This confirms that the FDP can be calibrated and used to determine the full Stokes vector of incident light over a broad spectral range. (2) $Q$ generally increases as $\phi$ increases, so that a higher angle of incidence is desirable. $\phi=70^{\circ}$ appears to be a good compromise because of the broad peak (discounting the dip) of high $Q$ at that angle.

Figure 3 shows the intensity reflectance $r$ of the $\mathrm{SiO}_{2}-\mathrm{Si}$ system for unpolarized or circularly polarized light as a function of the wavelength $\lambda$ at the same angles of incidence ( $\phi=$ $50^{\circ}, 60^{\circ}, 70^{\circ}, 80^{\circ}$ ). The reflectance level of $>1 / 3$ over the entire spectral range is adequate for the broadband operation of the FDP. Note that the region of low $Q$ in the near UV in Fig. 2 is one of high reflectance in Fig. 3.

Let us now consider the effect on the FDP of using a thicker (e.g., 200-nm) oxide layer on each detector surface. Figure 4 shows $Q(\lambda)$ versus $\lambda$ in this case. The oscillatory behavior of $Q$ (owing to interference in the oxide film) causes $Q$ to be zero and the instrument matrix to be singular at several discrete wavelengths. For completeness, Fig. 5 shows the associated spectral reflectance. In this case $r$ dips below $1 / 3$ at longer wavelengths, indicating another problem with thicker films.

The most important conclusion of this section is that, for broadband spectral application of the FDP with Si detectors (similar conclusions would hold for other types of detector, such as $\mathrm{Ge}$ in the IR), the thinnest dielectric $\left(\mathrm{SiO}_{2}\right)$ layer required for passivation is the most suitable optically, because this avoids interference-induced singularities of the instrument matrix.

\section{CHOICE OF LIGHT PATH}

As is noted in Section 3, the planes of incidence for each pair of successive reflections from detectors $D_{0}, D_{1}$, and $D_{2}$ (Fig. 1) should be neither coincident nor orthogonal (i.e., $\alpha_{1} \alpha_{2} \neq$ $\left.0,90^{\circ}, 180^{\circ}\right)$. This constraint on the light path is by far the most important. We have also shown that the choice of $\alpha_{1}$, $\alpha_{2}= \pm 45^{\circ}$ or $\pm 135^{\circ}$ is optimal; furthermore, this choice greatly simplifies the analytical expression of the instrument matrix, as can be verified easily by setting $\cos 2 \alpha_{1}=$ $\cos 2 \alpha_{2}=0$ and $\sin 2 \alpha_{1}=\sin 2 \alpha_{2}= \pm 1$ in Eqs. (11). We also noted in Section 7 that it is desirable to have large enough $\left(60^{\circ}-80^{\circ}\right)$ angles of incidence $\left(\phi_{0}, \phi_{1}\right.$, and $\left.\phi_{2}\right)$ at the detector surfaces.

Let the light path be denoted by $I O P Q R$ (Fig. 6), where $I O$ is the original direction of the incident light beam and $\mathrm{O}, \mathrm{P}$, $Q$, and $R$ are the points of refletion at which the light beam intercepts the surfaces of detectors $D_{0}, D_{1}, D_{2}$, and $D_{3}$, respectively. It should be noted that the lengths of the beam segments $O P, P Q$, and $Q R$ have no bearing on the operation of the FDP, in principle. Assume that the angles of incidence $\phi_{0}, \phi_{1}$, and $\phi_{2}$ (at $\mathrm{O}, \mathrm{P}$, and $\mathrm{Q}$, respectively) are specified. The first plane of incidence $I O P$ is fixed and can be taken as a reference plane (the $x y$ plane in Fig. 6). Specifying $\phi_{1}$ alone makes the second internal light segment $P Q$ as any one of the infinite number of generators of a right circular cone with an apex $\mathrm{P}$, an axis $O P$, and a semiapex angle of $180^{\circ}-2 \phi_{1}$. $\mathrm{Q}$ is fixed by $\alpha_{1}$. If $\alpha_{1}= \pm 45^{\circ}$ and $\alpha_{1}= \pm 135^{\circ}$ are considered equally good (which they are), we have a fourfold choice of the segment $P Q$. Likewise, for a given $\phi_{2}$,

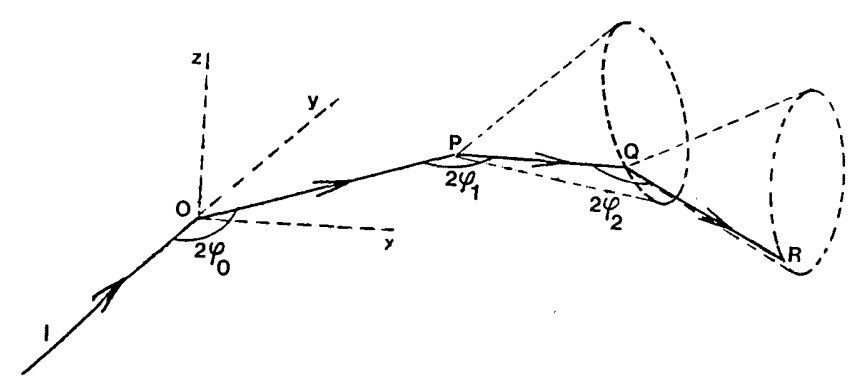

Fig. 6. A general light path $I O P Q R$ in the FDP. The meaning of the two indicated cones appears in the text. 


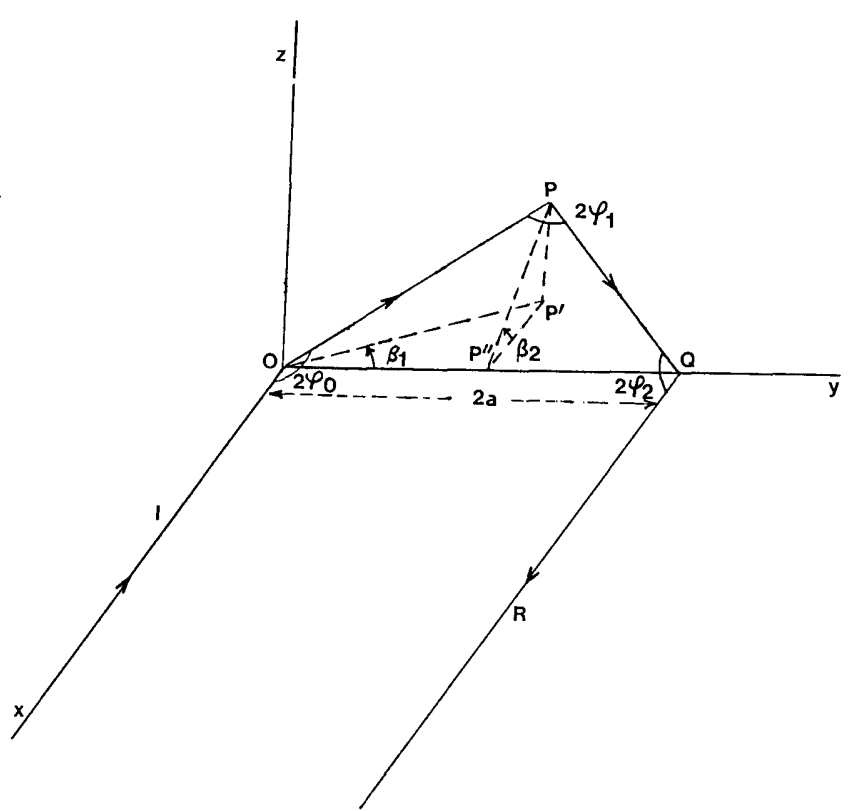

Fig. 7. A simple symmetrical light path $I O P Q R$ for the FDP. This path is determined completely by the distance $2 a$ and the two angles $\beta_{1}$ and $\beta_{2}$.

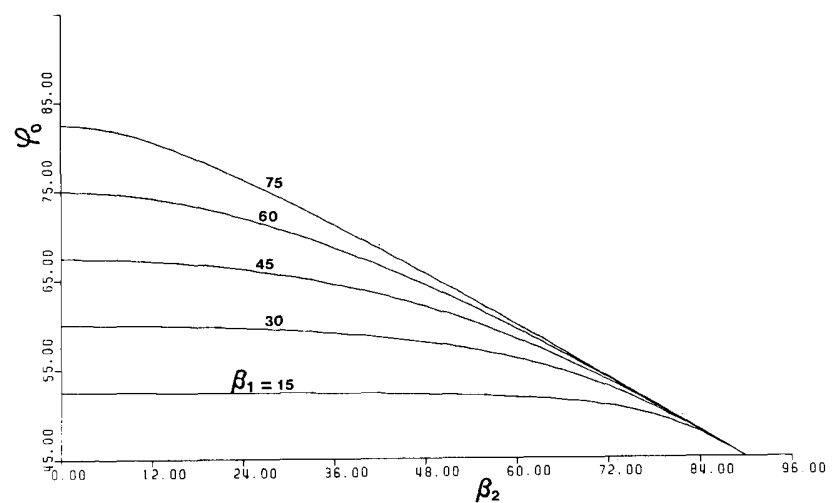

Fig. 8. Angle of incidence $\phi_{0}=\phi_{2}$ at the first and third detectors (positioned at $O$ and $Q$ ) for the light path of Fig. 7 plotted as a function of the angle $\beta_{2}$, with $\beta_{1}$ as a parameter, where $\beta_{1}=15^{\circ}, 30^{\circ}$, $45^{\circ}, 60^{\circ}, 75^{\circ}$, as marked by each curve.

$Q R$ is any one of the generators of another right circular cone with an apex at $Q$ and an axis $P Q$. Again, $Q R$ is fixed by $\alpha_{2}$. If $\alpha_{2}= \pm 45^{\circ}$ or $\pm 135^{\circ}$, we have another fourfold selection of the last light beam segment $Q R$. It follows that, for given $\phi_{0}$, $\phi_{1}$, and $\phi_{2}$ and for $\alpha_{1}, \alpha_{2}= \pm 45^{\circ}$ or $\pm 135^{\circ}$, there are 16 possible and optically equivalent light paths for the FDP.

Other criteria that may be used in selecting a light path are simplicity (or symmetry) and compactness. One path that satisfies these requirements is shown in Fig. 7. In this path the initial and final directions of the beam, $I O$ and $Q R$, are (anti) parallel, a distance $2 a$ apart, and in the $x y$ plane of a reference $x y z$ coordinate system (IO is in the direction of the negative $x$ axis). $\mathrm{O}$ and $\mathrm{Q}$ lie on the $y$ axis, and $\mathrm{P}$ is in the plane $y=a$, which is the plane of mirror-image symmetry for this light path. $\mathrm{P}^{\prime}$ is the foot of the normal from $\mathrm{P}$ to the $x y$ plane, and $\mathrm{P}^{\prime \prime}$ is the foot of the normal from $\mathrm{P}$ to the $y$ axis. Because of symmetry, we have

$$
\phi_{0}=\phi_{2}, \quad \alpha_{1}=\alpha_{2} .
$$

The angles of incidence $\phi_{0}\left(=\phi_{2}\right)$ and $\phi_{1}$ and the rotation angle of the plane of incidence $\alpha_{1}\left(=\alpha_{2}\right)$ are all determined by two characteristic angles $\beta_{1}$ and $\beta_{2}$ that are defined in Fig. 7. By introducing a set of unit vectors along the segmented light path and performing a simple vector analysis, we obtain

$$
\begin{aligned}
\cos 2 \phi_{0} & =-\tan \beta_{1} /\left(1+\tan ^{2} \beta_{1} \sec ^{2} \beta_{2}\right)^{1 / 2} \\
\cos 2 \phi_{1} & =\left(-1+\tan ^{2} \beta_{1} \sec ^{2} \beta_{2}\right) /\left(1+\tan ^{2} \beta_{1} \sec ^{2} \beta_{2}\right), \\
\cos \alpha_{1} & =\cos \beta_{2} /\left(1+\tan ^{2} \beta_{1} \tan ^{2} \beta_{2}\right)^{1 / 2}
\end{aligned}
$$

Notice that the only dimensional parameter, $2 a$, is not needed to define the essential angles of the light path.

Figures 8, 9, and 10 show $\phi_{0}, \phi_{1}$, and $\alpha_{1}$, respectively, plotted as functions of $\beta_{2}$ at constant values of $\beta_{1}=15^{\circ}, 30^{\circ}$, $45^{\circ}, 60^{\circ}, 75^{\circ}$.

To cite a specific example, we take a light path with $\beta_{1}=$ $15^{\circ}, \beta_{2}=42^{\circ}$, giving $\phi_{0}=52.30^{\circ}, \phi_{1}=70.17^{\circ}$, and $\alpha_{1}=43.75^{\circ}$ from Eqs. (38). For identical Si detectors with thin (10-nm) oxide layers, $\phi_{1}=70.17^{\circ}$ is a good angle, because $\sin \Delta_{1}>0.4$ and $\sin 4 \psi_{1}>0.34$ over the entire 1.5-6-eV spectral range. With $\phi_{0}=\phi_{2}=52.30^{\circ}, \psi_{0}=\psi_{2}$, and the combined psi factor for detectors $\mathrm{D}_{0}$ and $\mathrm{D}_{2}$ in the determinant of the instrument matrix becomes proportional to $\left(\sin 4 \psi_{0}\right)^{2}$, which is $>0.09$ over the same spectral range. Finally, the alpha factor of $\operatorname{det} \mathbf{A}$ in Eq. $(21)$ is $\sin ^{2}\left(2 \times 43.75^{\circ}\right) \cong 0.998$, which is

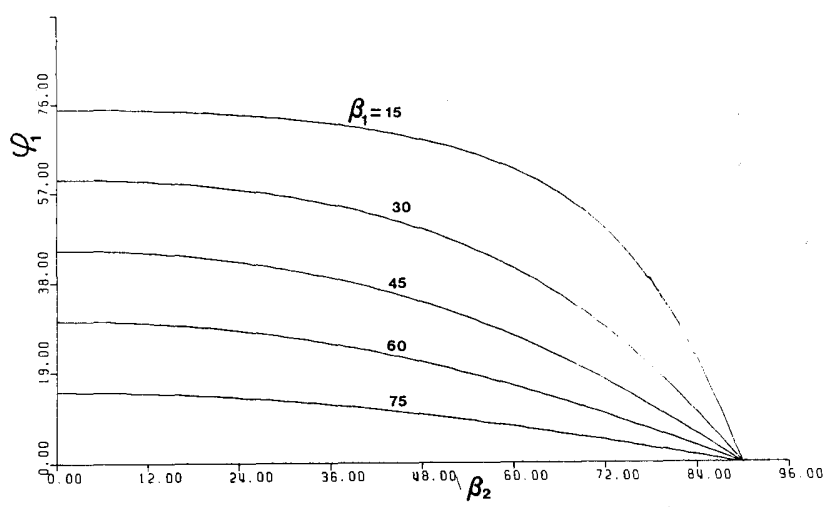

Fig. 9. Angle of incidence $\phi_{1}$ at the second detector (positioned at P) for the light path of Fig. 7 plotted as a function of $\beta_{2}$ with $\beta_{1}=15^{\circ}$, $30^{\circ}, 45^{\circ}, 60^{\circ}, 75^{\circ}$, as marked by each curve.

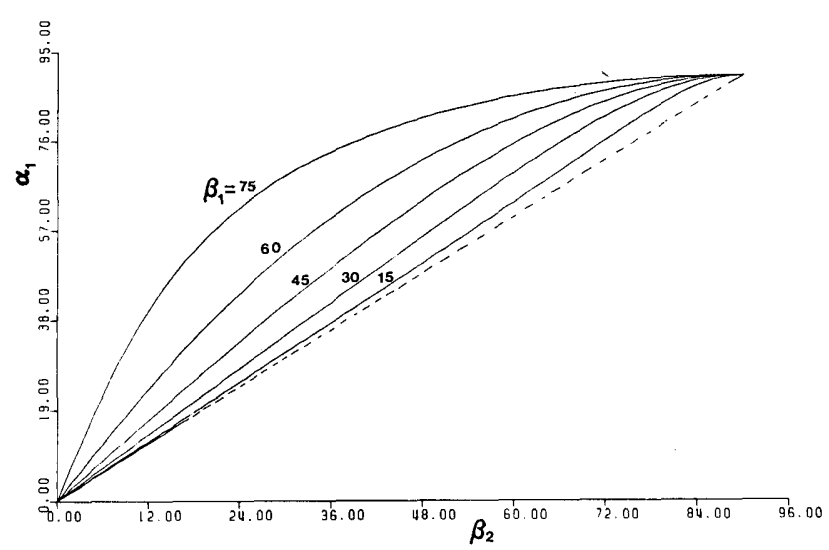

Fig. 10. Rotation angle $\alpha_{1}=\alpha_{2}$ between successive planes of incidence for the light path of Fig. 7 plotted versus the angle $\beta_{2}$ with $\beta_{1}=$ $15^{\circ}, 30^{\circ}, 45^{\circ}, 60^{\circ}, 75^{\circ}$, as marked by each curve. 
negligibly less than the maximum value of 1 . This numerical example confirms that a simple compact light path such as that shown in Fig. 7 (with $\beta_{1}=15^{\circ}$ and $\beta_{2}=42^{\circ}$ ) is well suited for the FDP. In fact, the first operated FDP ${ }^{10}$ used a similar light path.

\section{DETERMINING THE INSTRUMENT MATRIX A BY CALIBRATION}

For a given light path and photodetectors with well-characterized surfaces (e.g., $\mathrm{SiO}_{2}$-coated $\mathrm{Si}$ ), and known responsivities, the instrument matrix $\mathbf{A}(\lambda)$ can be calculated as a function of the wavelength $\lambda$, as is described in Section 2 . An alternative, more-practical approach is to determine $\mathbf{A}$ by calibration, using a polarized-light source. By recording the output current vectors $\mathbf{I}_{m}$ of the FDP, at each $\lambda$, that correspond to four different input polarizations, described by four linearly independent Stokes vectors $\mathbf{S}_{m}(m=1,2,3$, 4), Eq. (1) can be written four times, and the results can be compressed into one matrix equation,

$$
\mathcal{J}=\mathcal{A} S
$$

where

$$
\begin{aligned}
& \boldsymbol{J}=\left[\begin{array}{llll}
\mathbf{I}_{1} & \mathbf{I}_{2} & \mathbf{I}_{3} & \mathbf{I}_{4}
\end{array}\right] \text {, }
\end{aligned}
$$

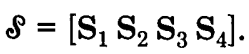

From Eq. (39) we obtain

$$
\mathbf{A}=\mathcal{J} \mathcal{\rho}^{-1}
$$

Notice that $\rho^{-1}$ exists because $\mathbf{S}_{m}$ in Eq. (40b) are linearly independent Stokes vectors.

Equation (41) offers the basic prescription for the experimental measurement of $\mathbf{A}$. Once $\mathbf{A}$ has been determined by calibration, the FDP becomes ready to accept and to measure any unknown polarization of light of same wavelength as the calibration wavelength.

If we take the determinant of both sides of Eq. (41), we obtain

$$
\operatorname{det} \mathbf{A}=\operatorname{det} \mathcal{J} / \operatorname{det} \mathcal{S} \text {. }
$$

Because $\operatorname{det} \delta \neq 0$ by the choice of calibration states, it follows that we must have

$$
\operatorname{det} \mathcal{J} \neq 0
$$

if $\mathbf{A}$ is to be nonsingular. Relation (43) leads to the following simple and important practical theorem:

At a given wavelength, if the four output current vectors of the FDP, $\mathbf{I}_{m}(m=1,2,3,4)$, that are generated by four linearly independent input polarization states are themselves linearly independent, the instrument matrix $\mathbf{A}$ is nonsingular, and the FDP is capable of determining the full Stokes vector of input light of any state of polarization.

If the four calibration states are represented by four points on the surface of the Poincare sphere, ${ }^{11}$ an optimum choice results if the four points are as far apart as possible, which makes them the vertices of a tetrahedron. This is the maximum-volume pyramid inscribed inside the sphere and corresponds to maximum determinant of $\delta$. One such selection is indicated in Fig. 11, in which the calibration states $\mathrm{C}_{2}$, $\mathrm{C}_{3}$, and $\mathrm{C}_{4}$ are the vertices of an equilateral triangle inscribed inside a circle with a latitude that is a distance of $1 / 3$ below

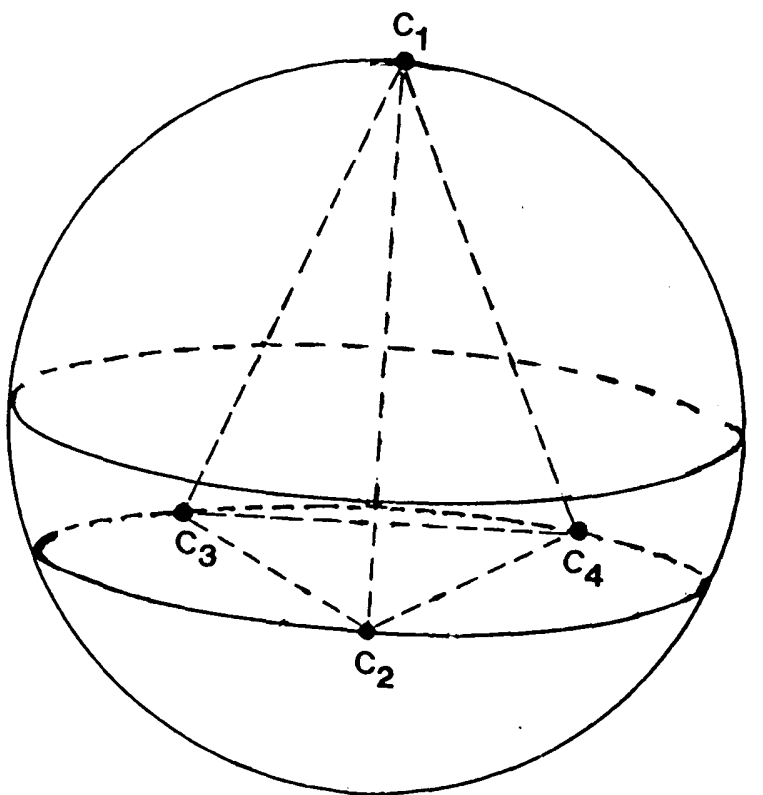

Fig. 11. The optimum calibration polarization states $\mathrm{C}_{1}, \mathrm{C}_{2}, \mathrm{C}_{3}$, and $\mathrm{C}_{4}$ are the vertices of a tetrahedron (maximum-volume pyramid) inscribed inside the Poincaré sphere.

the plane of the equator of the unit-radius Poincare sphere. $\mathrm{C}_{2}, \mathrm{C}_{3}$, and $\mathrm{C}_{4}$ represent left-handed elliptical polarization states of the same ellipticity $\left(\epsilon=-9.736^{\circ}\right.$ and the ellipse axial ratio $|\tan \epsilon|=0.1716)$ that are $60^{\circ}$ apart in azimuth. The remaining calibration state $C_{1}$ is the fourth vertex of the tetrahedron and represents the right circular polarization.

From simple geometry, the maximum tetrahedron volume is

$$
V_{\max }=8 \sqrt{3} / 27
$$

Consider, for comparison, the previously suggested ${ }^{12}$ set of calibration states that consist of three linear polarizations $45^{\circ}$ apart in azimuth and the right or left circular polarization state. The volume of the associated pyramid inside the Poincaré sphere is $V=1 / 3$; hence

$$
V_{\max } / V=8 \sqrt{3} / 9=1.54
$$

is the relative improvement factor that results from choosing the optimal set of calibration states.

The calibration polarization states can be produced by passing a monochromatic collimated source beam through a linear polarizer followed by a quarter-wave retarder of adjustable azimuths. Rotation of these elements around the beam as an axis generates all possible totally polarized states. $^{13}$ By providing two pinned relative positions (at $-9.736^{\circ}$ and $45^{\circ}$ ) of the retarder fast axis with respect to the polarizer transmission axis, the three optimal elliptical calibration states can be obtained easily by two successive $60^{\circ}$ rotations of the two elements as one unit in their $-9.736^{\circ}$ relative position, followed by change of the relative position to $45^{\circ}$ to produce the circular state.

\section{SUMMARY}

In this paper we present a general analysis of the FDP. In Section 2, we obtain an explicit expression of the instrument matrix $\mathbf{A}$ in terms of the reflection properties of the surfaces 
of the first three detectors, $r_{m}, \psi_{m}, \Delta_{m}(m=0,1,2)$, the angles of rotation $\alpha_{1}$ and $\alpha_{2}$ of the plane of incidence between successive reflections, and the responsivities $k_{m}(m=0,1,2$, 3 ) of all detectors. In general, $\mathbf{A}$ is nonsingular and $\operatorname{det} \mathbf{A} \neq$ 0 if the light beam does not remain in one plane. The conditions for the existence of occasional singularities, $\operatorname{det} \mathbf{A}$ $=0$, are considered in detail in Section 3 . Only one differential reflection plane shift, $\Delta_{1}$, is important; $\Delta_{0}$ and $\Delta_{2}$ of the first and third detectors do not appear in the expression for $\operatorname{det} \mathbf{A}$ in Eq. (21), and hence they are unimportant.

In Section 4, optimum values of the surface parameters $\psi_{0}$, $\psi_{1}, \Delta_{1}$, and $\psi_{2}$ and of the rotation angles $\alpha_{1}$ and $\alpha_{2}$ are determined that maximize $|\operatorname{det} \mathbf{A}|$ and hence make $\mathbf{A}$ as far from singular as possible. In Section 5 we offer two criteria for the selection of detector surface reflectances $r_{0}, r_{1}$, and $r_{2}$ that determine the partition of energy among the four detectors.

It is advantageous to tilt the last detector by a small angle to avoid multiple reflections between detectors, and in Section 6 we prove that this has no significant effect on the FDP.

Operation of the FDP over a broadband spectral range is discussed in Section 7. Taking, as an example, identical Si detectors $D_{0}, D_{1}$, and $D_{2}$; equal incidence angles; and optimum rotation angles $\left( \pm 45^{\circ}\right.$ or $\left.\pm 135^{\circ}\right)$, we show that the instrument matrix $\mathbf{A}$ is nonsingular over the entire $1.5-6-\mathrm{eV}$ $(\sim 200-800 \mathrm{~nm})$ spectral range when the Si surfaces are coated by only a thin (10-nm) $\mathrm{SiO}_{2}$ passivation layer. Thicker films (e.g., $200 \mathrm{~nm}$ ) lead to interference-induced singularities of $\mathbf{A}$ at discrete wavelengths in the same spectral interval.

Provided that successive planes of incidence are neither coincident nor orthogonal, there is considerable flexibility in the choice of a light path, as is discussed in Section 8.

Finally, Section 9 we provide an account of how $\mathbf{A}$ can be measured by calibration. For a given black box of four detectors, we present a simple practical test for determining whether $\operatorname{det} \mathbf{A}$ is nonsingular. If the calibration light beam is sequentially polarized in four linearly independent polarization states, represented by four points on the Poincaré sphere $\mathrm{C}_{1}, \mathrm{C}_{2}, \mathrm{C}_{3}$, and $\mathrm{C}_{4}$, which are not in one plane, and the corresponding four output current vectors $\mathbf{I}_{m}(m=1,2,3,4)$ of the FDP are found to be also linearly independent (i.e., $\operatorname{det}\left[\mathbf{I}_{1}, \mathbf{I}_{2} \mathbf{I}_{3} \mathbf{I}_{4}\right] \neq 0$ ), then $\operatorname{det} \mathbf{A} \neq 0$. An optimum choice of calibration states occurs when $\mathrm{C}_{1}, \mathrm{C}_{2}, \mathrm{C}_{3}$, and $\mathrm{C}_{4}$ become the vertices of a tetrahedron (maximum-volume pyramid) inscribed inside the Poincaré sphere.

\section{ACKNOWLEDGMENTS}

This research is supported by the National Science Foundation under grant no. ECS852003. This paper was presented at the 1987 Annual Meeting of the Optical Society of America in Rochester; New York, October 18-23, 1987.

\section{REFERENCES}

1. See, for example, M. Born and E. Wolf, Principles of Optics (Pergamon, New York, 1975), p. 554.

2. R. M. A. Azzam, "Arrangement of four photodetectors for measuring the state of polarization of light," Opt. Lett. 10, 309-311 (1985); U.S. Patent 4,681,450 (July 21, 1987).

3. R. M. A. Azzam, E. Masetti, I. M. Elminyawi, and F. G. Grosz, "Construction, calibration, and testing of a four-detector photopolarimeter," Rev. Sci. Instrum. 59, 84-88 (1988).

4. R. M. A. Azzam and N. M. Bashara, Ellipsometry and Polarized Light (North-Holland, Amsterdam, 1987).

5. R. M. A. Azzam, "Contours of constant principal angle and constant principal azimuth in the complex $\epsilon$ plane," J. Opt. Soc. Am. 71, 1523-1528 (1981).

6. R. M. A. Azzam, "Stationary property of normal-incidence reflection from isotropic surfaces," J. Opt. Soc. Am. 72, 1187-1189 (1982).

7. R. M. A. Azzam, "In-line light-saving photopolarimeter and its fiber-optic analog," Opt. Lett. 12, 558-560 (1987).

8. A. J. Warnecke and P. J. LoPresti, "Refractive index dispersion in semiconductor-related thin films," IBM J. Res. Dev. 17, 256262 (1973).

9. D. E. Aspnes and A. A. Studna, "Dielectric functions and optical parameters of $\mathrm{Si}, \mathrm{Ge}, \mathrm{GaP}, \mathrm{GaAs}, \mathrm{GaSb}, \mathrm{InP}$, InAs, and InSb from 1.5 to $6.0 \mathrm{eV}$," Phys. Rev. B 27, 985-1009 (1983).

10. R. M. A. Azzam, E. Masetti, F. G. Grosz, and I. Elminyawi, "Four-detector photopolarimeter: first experimental results," J. Opt. Soc. Am. A 4(13), P78 (1987).

11. See, for example, Ref. 4, Sec. 1.8.

12. P. S. Hauge, "Mueller matrix ellipsometry with imperfect compensators," J. Opt. Soc. Am. 68, 1519-1528 (1978).

13. See, for example, Ref. 4, Sec. 3.9.1. 